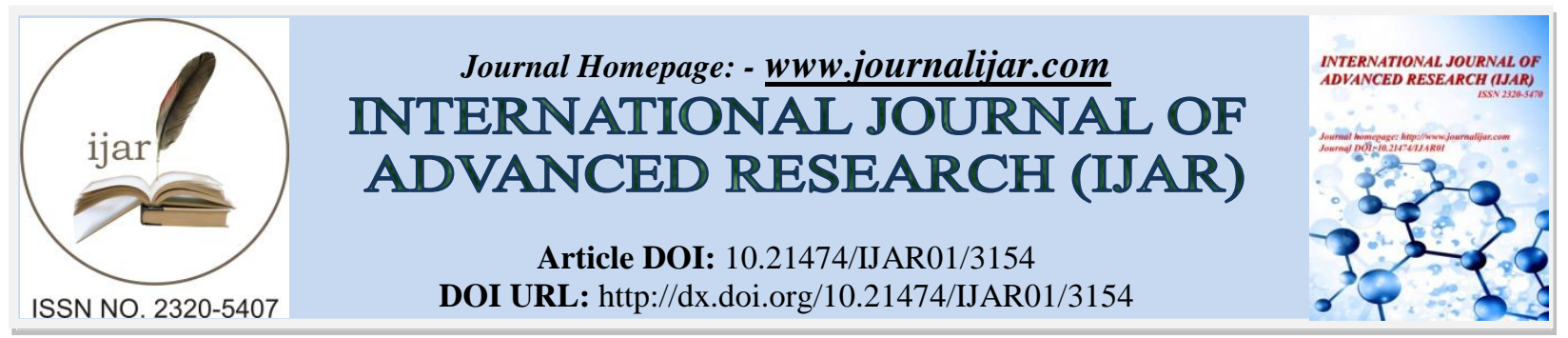

RESEARCH ARTICLE

\title{
GC GLOBULIN GENE POLYMORPHISM: A CANDIDATE LOCUS FOR SUSCEPTIBILITY TO RHEUMATOID AND OSTEOARTHRITIS.
}

1. Department of Genetics, Osmania University 500007.

Arshia Fatima $^{1 *}$, Mohd Ishaq ${ }^{1,2}$ and parveen ${ }^{2}$

2. Salar-E-Millat Research Centre for cellular and Molecular Medicine, PEH, DCMS, Hyderabad India.

\section{Manuscript Info}

Manuscript History

Received: 19 December 2016

Final Accepted: 10 January 2017

Published: February 2017

Key words:-

Gc globulin, Rheumatoid arthritis,

Osteoarthritis

\begin{abstract}
Multiple factors are believed to be involved in the manifestation of Rheumatoid arthritis and Osteoarthritis. Genetic factors are also considered as important predisposing factor in these debilitating conditions. In the present study we investigated the role of Group specific component also known as Gc globulin gene polymorphism in susceptibility to these frequently observed arthritic condition. Gc globulin gene polymorphism was investigated in 115 RA patients, 47 OA cases along with 100 healthy controls. The percentages for Gc 2-2, Gc 2-1 and Gc 1-1 were 8\%, 35\% and 57\% in the controls. Significantly increased frequency of Gc 2-1 heterozygotes was observed in RA $86.09 \%$ and OA $95.74 \%(* \mathrm{p}<0.05)$. It is inferred that the individuals with Gc 2-1 globulin have high risk of developing RA or OA compared to those with Gc 2-2 and Gc 1-1.
\end{abstract}

Copy Right, IJAR, 2017,. All rights reserved.

\section{Introduction:-}

Arthritis is pain, swelling and stiffness of joints. As it is known that joints are parts of the body where two bones are attached and usually lined with cartilage, a rubbery material that prevents the bones from rubbing together [1]. Arthritis can result from too much wear and tear on the joint or from illness that can cause inflammation of joints [2].

Rheumatoid arthritis involves inflammation of the joint lining called synovium, which leads to joint deterioration. It is a chronic disease characterized by general ill health and chronic inflammation of the tissue around the joints and tendons [3]. It presents with symmetrical, inflammatory and destructive joint changes and tenosynovitis, but is also associated with other systemic findings that can affect virtually any body tissue or organ [4].

Rheumatoid Arthritis is a frequently observed disorder of connective tissue and is an important cause of disability, morbidity and mortality [5]. Rheumatoid Arthritis occurs worldwide with variable incidence and severity. The etiology of Rheumatoid Arthritis remains unclear but there is evidence of genetic predisposition to the disease [6].

OA is another form of arthritis, especially among older obese people [7, 8]. It is the joint disease caused by the breakdown of cartilage that firm rubbery tissue that cushions bones at joints. Men and women are affected but symptoms occur earlier and appear to be more severe in women [9]. The exact etiology of OA is unknown but it is believed that multiple factors (e.g. heredity, trauma and obesity) interact to cause this disorder. The pathophysiology involves a combination of mechanical, cellular and biochemical processes [10]. The interaction of 
these processes leads to changes in the composition and mechanical properties of the particular cartilage. Cartilage is composed of water, collagen and it absorbs energy from the shock of physical movement. In OA cartilage breaks down and wears away. As a result, the bones rub together, causing pain, swelling and stiffness. OA may also limit the range of motion in affected individuals [11]. Most often, OA develops in the hands, knee, hips and spine [12].

Genetic factors play a important role in the etiology of RA and OA in addition to biochemical and immunological factors. Candidate gene approach was employed using Gc globulin to investigate disease association in RA and OA patients.

The vitamin D binding protein (DBP) also known as Gc globulin is a multifunctional plasma protein that can bind several diverse ligands. It is a member of the albumin and alpha-fetoprotein gene family and shares considerable amino acid homology with this protein.

Gc is a 52 to $58 \mathrm{kDa}$ multifunctional plasma protein, synthesized mainly by hepatocytes. Polymorphism in the Gc gene (Codminant alleles) gives rise to three major electrophoretic variants of Gc (Gc2, Gc1s and Gc1f) which differ by amino acid substitution as well as glycosylation [13]. They differ with regards to relative vitamin binding capacity. Reduced vitamin D binding capacity and reduced vitamin D levels are believed to be associated with susceptibility to autoimmunity as well as reduced bone density in RA and OA patients. The physiological significance related to the various phenotypes is yet to be discovered.

Gc is the major carrier protein of vitamin D and its metabolites in the circulation and is important for preservation of the Vitamin [14]. Gc globulin also transports components such as fatty acids and endotoxin and it is an important player in the actin scavenging system [15]. Gc binds actin released from cells upon injury and Gc actin complexes are rapidly cleared from the circulation, thereby preventing the harmful effects of actin filaments in blood vessels. The resulting decrease in Gc concentration makes Gc usable as a prognostic indicator of survival of patients with significant tissue injury after trauma and among patients with hepatic failure [16]. The vitamin D binding protein (DBP) is the major carrier protein for vitamin D metabolites in plasma. Polymorphisms in DBP have been described to be associated with an increased bone fracture risk and diabetes.

High frequency of the GC*2 allele and a low frequency of the $\mathrm{GC} * 1 \mathrm{~S}$ allele were observed in rheumatoid arthritis and osteoarthritis compared to controls [17]. There are few studies from India on role of Gc globulin polymorphism in susceptibility to RA and OA. Hence the present study was carried to investigate the role of polymorphic variants of Gc globulin in RA and OA.

\section{Material and Methods:-}

The blood samples of RA and OA patients were collected from local Rheumatology centre in the Hyderabad under the guidance of a Rheumatologist. The control samples were randomly collected from general population.

Protein polymorphism of Gc globulin was determined in the blood samples of RA and OA patients by polyacrylamide gel electrophoresis and gels were stained by amidoblack.The method followed for electrophoresis and phenotyping was according to procedure described in Giblet 1961 [18].

Table 1:- Distribution of Gc Phenotypes in RA and OA cases

\begin{tabular}{|l|l|l|l|l|}
\hline Category & Total & Gc 2-2 & Gc 2-1 & Gc 1-1 \\
\hline RA & 115 & $2(1.74 \%)$ & $99(86.09 \%)^{*}$ & $1(12.17 \%)^{*}$ \\
\hline OA & 47 & $1(2.12 \%)$ & $45(95.74 \%)^{*}$ & $1(2.12 \%)^{*}$ \\
\hline Control & 100 & $8(8.00 \%)$ & $35(35.00 \%)$ & $57(57.00 \%)$ \\
\hline
\end{tabular}

$* \mathrm{p}<0.05$

Table 2:- Distribution of Gc Phenotypes in RA and OApatients with respect to sex *p<0.05

\begin{tabular}{|l|l|l|l|l|}
\hline Category & Total & Gc 2-2 & Gc 2-1 & Gc 1-1 \\
\hline RA & & & & \\
Male & 21 & $0(0.00 \%)$ & $19(90.47 \%)$ & $2(9.53 \%)$ \\
Female & 94 & $1(2.12 \%)^{*}$ & $80(85.11 \%)$ & $12(12.76 \%)$ \\
\hline OA & & & & $1(12.50 \%)$ \\
Male & 8 & $0(0.00 \%)$ & $7(87.50 \%)$ & $12(0.00 \%)$ \\
Female & 39 & $1(2.56 \%)$ & $3(97.44 \%)$ & \\
\hline
\end{tabular}




\section{Result and Discussion:-}

A perusal of Table 1 reveals significantly high frequency of Gc 2-1 in both RA and OA patients compared with controls $\quad(86.09 \%$ in RA and $95.74 \%$ in OA $(* \mathrm{p}<0.05)$ and only $35 \%$ in controls). Relative risk analysis by odds ratio revealed that patients with Gc 2-1 have increased risk of developing RA and OA. In RA relative risk was found to be 11.49 while in OA it was 41.78. These results indicate that gene coding Gc globulin can be considered as potential candidate gene for RA and OA. The susceptibility is found to be higher even in OA than in RA patients. Distribution of Gc phenotype in male and female revealed that both male and female cases with Gc 2-1 phenotype are significantly higher than in the control samples.

\section{References:-}

1. Tam LS et al. (2016 ): The Role of High-resolution Peripheral Quantitative Computed Tomography as a Biomarker for Joint Damage in InflammatoryArthritis. J Rheumatol., 43(10):1911-1913.

2. Bhattaram P, Chandrasekharan U. et al (2016): The joint synovium: A critical determinant of articular cartilage fate in inflammatory joint diseases. Semin Cell Dev Biol., pii: S1084-9521(16)30135-5. doi: 10.1016/j.semcdb.2016.05.009.

3. Catrina AI, Joshua Vet al. (2016): Mechanisms involved in triggering rheumatoid arthritis. IImmunol Rev., 269(1):162-74. doi: 10.1111/imr.12379.

4. Kim-Howard XR, Staudt L et al. (2005): Update in rheumatoid arthritis therapy. J Okla State Med Assoc., 98(2):53-62. Review

5. Mercan R et al.(2016):The Association Between Neutrophil/Lymphocyte Ratio and Disease Activity in Rheumatoid Arthritis and Ankylosing Spondylitis. J Clin Lab Anal., 30(5):597-601. doi: 10.1002/jcla.21908.

6. Zerbini CA. et al. (2016): IOF Chronic Inflammation and Bone Structure (CIBS) Working Group Biologic therapies and bone loss in rheumatoid arthritis. Osteoporos Int.,

7. Wen Let al.(2016) : Associations between body composition measurements of obesity and radiographic osteoarthritis in older adults: Data from the Dong-gu Study. BMC Musculoskelet Disord., 17:192. doi: 10.1186/s12891-016-1040-9.

8. Reyes C, Leyland KM, et al. (2016): Association Between Overweight and Obesity and Risk of Clinically Diagnosed Knee, Hip, and Hand Osteoarthritis: A Population-Based Cohort Study. Arthritis Rheumatol., 68(8):1869-75. doi: 10.1002/art.39707.

9. Pal CP, Singh P, et al. (2016): Epidemiology of knee osteoarthritis in India and related factors. Indian J Orthop., 50(5):518-522.

10. Martel-Pelletier J, Barr AJ. et al. (2016):Osteoarthritis. Nat Rev Dis Primers., 13;2:16072. doi: 10.1038/nrdp.2016.72.

11. Sharif B, Garner R, et al. (2016): Risk of work loss due to illness or disability in patients with osteoarthritis: a population-based cohort study. Rheumatology (Oxford)., 55(5):861-8. doi: 10.1093/rheumatology/kev428.

12. Prieto-Alhambra D, Judge A, et al. (2014): Incidence and risk factors for clinically diagnosed knee, hip and hand osteoarthritis: influences of age, gender and osteoarthritis affecting other joints. Ann Rheum Dis., 2014 Sep; 73(9):1659-64. doi: 10.1136/annrheumdis-2013-203355.

13. Braun A, Bichlmaier R, et al. (1992): Molecular analysis of the gene for the human vitamin-D-binding protein (group-specific component): allelic differences of the common genetic GC types. Hum Genet., 89(4):401-6.

14. Safadi FF, Thornton P, et al. (1999): Osteopathy and resistance to vitamin D toxicity in mice null for vitamin D binding protein. J Clin Invest., 103(2):239-51.

15. Lee WM et al. (1992): Galbraith RM The extracellular actin-scavenger system and actin toxicity. N Engl J Med., 14;326(20):1335-41.

16. Dahl B, Schiødt FV, et al. (1998) :Serum Gc-globulin in the early course of multiple trauma. Crit Care Med., 26(2):285-9

17. Kahl LE, Kamboh MI, et al. (1989):Alpha-1-antitrypsin (PI) and vitamin-D binding globulin (GC) phenotypes in rheumatoid arthritis: absence of an association. Dis Markers., 7(2):71-8.

18. Giblett ER et al. (1961): Genetic markers in human blood. Blackwell scientific publisher Oxford., $78: 723$ 